\title{
PRETEXT Stage 2
}

National Cancer Institute

\section{Source}

National Cancer Institute. PRETEXT Stage 2. NCI Thesaurus. Code C28073.

Hepatoblastoma Pretreatment Extent of Disease System stage in which tumor involves two adjoining quadrants, while the other two adjoining liver quadrants are free of tumor. $-2004$ 